Anais da Academia Brasileira de Ciências (2014) 86(1): 3-9

(Annals of the Brazilian Academy of Sciences)

Printed version ISSN 0001-3765 / Online version ISSN 1678-2690

http://dx.doi.org/10.1590/0001-37652014107412

www.scielo.br/aabc

\title{
New classes of spatial central configurations for the 7-body problem
}

\author{
ANTONIO CARLOS FERNANDES and LUIS FERNANDO MELLO
}

Instituto de Matemática e Computação, Universidade Federal de Itajubá, Avenida BPS, 1303, Pinheirinho, 37500-903 Itajubá, MG, Brasil

Manuscript received on July 24, 2012; accepted for publication on May 6, 2013

\begin{abstract}
In this paper we show the existence of new families of spatial central configurations for the 7-body problem. In the studied spatial central configurations, six bodies are at the vertices of two equilateral triangles $\mathcal{T}_{1}$, $\mathcal{T}_{2}$ and one body is located out of the parallel distinct planes containing $\mathcal{T}_{1}$ and $\mathcal{T}_{2}$. The results have simple and analytic proofs.
\end{abstract}

Key words: Spatial central configuration, 7-body problem, stacked central configuration, central configuration.

\section{INTRODUCTION}

In this paper we study spatial central configurations for the $N$-body problem. Before we can address our problem, some definitions are in order. Consider $N$ punctual bodies with masses $m_{i}>0$ located at the points $r_{i}$ of the Euclidean space $\mathbb{R}^{3}$ for $i=1, \ldots, N$. Assume that the origin of the inertial system is the center of mass of the system (inertial barycentric system). The set $\left\{\left(r_{1}, r_{2}, \ldots, r_{N}\right) \in \mathbb{R}^{3 N}: r_{i} \neq r_{j}, i \neq j\right\}$ is called space of configurations.

For the Newtonian $N$-body problem a configuration of the system is central if the acceleration of each body is proportional to its position relative to the inertial barycentric system. It is usual to study classes of central configurations modulo dilations and rotations. See Hagihara (1970), Moeckel (1990), Saari (1980), Smale (1970), Wintner (1941) and references therein for more details.

Spatial central configurations give rise to homothetic orbital motions which are the simplest solutions of the $\mathrm{N}$-body problem. However to know the central configurations for a given set of bodies with positive masses is a very hard and unsolved problem even in the case of few bodies. For instance, in Lehmann-Filhés (1891) and Wintner (1941) can be found classical examples of spatial central configurations where the bodies with suitable masses are at the vertices of a regular tetrahedron and a regular octahedron, respectively. More recent examples were studied in Corbera and Llibre (2008) and Corbera and Llibre (2009) in which $2 N$ and $3 N$ bodies are arranged at the vertices of two and three nested regular polyhedra, respectively. See also Zhu (2005) in which nested regular tetrahedrons were studied.

A stacked spatial central configuration is defined as a central configuration for the spatial $N$-body problem where a proper subset of the $N$ bodies is already in a central configuration. See Hampton and Santoprete (2007), Mello and Fernandes (2011a, b), Mello et. al. (2009) and Zhang and Zhou (2001).

AMS Classification: Primary 70F10, 70F15, 37N05.

Correspondence to: Luis Fernando Mello

E-mail:1fmelo@unifei.edu.br 


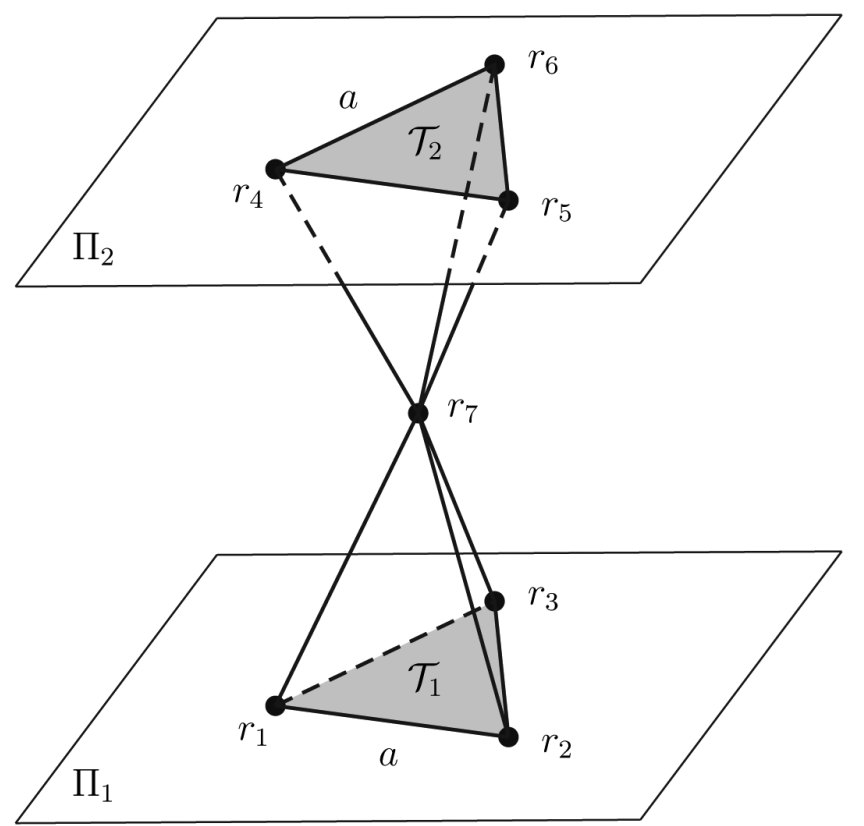

Figure 1 - Illustration of the configurations studied here. The position vectors $r_{1}, r_{2}$ and $r_{3}$ are at the vertices of an equilateral triangle $\mathcal{T}_{1}$ whose sides have length $a>0, r_{4}, r_{5}$ and $r_{6}$ are at the vertices of an equilateral triangle $\mathcal{T}_{2}$ whose sides have length $a>0$ and $r_{7}$ is out of the parallel distinct planes $\Pi_{1}$ and $\Pi_{2}$ that contain $\mathcal{T}_{1}$ and $\mathcal{T}_{2}$, respectively.

Denote by $r_{i j}=\left|r_{i}-r_{j}\right|$ the Euclidean distance between the bodies at $r_{i}$ and $r_{j}$. The main results of this paper are the following.

Theorem 1. Consider 7 bodies with masses $m_{1}, m_{2}, \ldots, m_{7}$, located according to the following description (see Figure 1):

(i) The position vectors $r_{1}, r_{2}$ and $r_{3}$ are at the vertices of an equilateral triangle $\mathcal{T}_{1}$ whose sides have length $a>0$;

(ii) The position vectors $r_{4}, r_{5}$ and $r_{6}$ are at the vertices of an equilateral triangle $\mathcal{T}_{2}$ whose sides have length $a>0$;

(iii) The triangles $\mathcal{T}_{1}$ and $\mathcal{T}_{2}$ belong to parallel distinct planes $\Pi_{1}$ and $\Pi_{2}$, respectively;

(iv) The triangles $\mathcal{T}_{1}$ and $\mathcal{T}_{2}$ are coincident under translation;

(v) The position vector $r_{7}$ is located between the planes $\Pi_{1}$ and $\Pi_{2}$. Then the following statements hold.

Then the following statements hold.

(a) If $r_{i 7}=d>0$ for all $i \in\{1,2, \ldots, 6\}$, then in order to have a central configuration the masses must satisfy:

$$
m_{1}=m_{2}=m_{3}=m_{4}=m_{5}=m_{6} .
$$

(b) If $r_{i 7}=d>0$ for all $i \in\{1,2, \ldots, 6\}$ and $m=m_{1}=m_{2}=m_{3}=m_{4}=m_{5}=m_{6}$, then there is only one class of central configuration.

Furthermore such central configurations are independent of the values of the masses $m_{7}$ and $m$ and are of stacked type.

The proof of Theorem 1 is given in the next section. Concluding remarks are presented in Section 3. 


\section{PROOF OF THEOREM 1}

According to our assumptions, the equations of motion of the $N$ bodies are given by Newton (1687)

$$
\ddot{r}_{i}=-\sum_{j=1, j \neq i}^{N} \frac{m_{j}}{r_{i j}^{3}}\left(r_{i}-r_{j}\right),
$$

for $i=1, \ldots, N$. Here the gravitational constant is taken equal to one. Equations (1) are well defined since $r_{i j} \neq 0$ for $i \neq j$.

Note that to find central configurations is essentially an algebraic problem. In fact, from the definition of central configuration there exists $\lambda \neq 0$ such that $\ddot{r}_{i}=\lambda r_{i}$, for all $i=1, \ldots, N$. From equation (1) it follows that

$$
\lambda r_{i}=-\sum_{j=1, j \neq i}^{N} \frac{m_{j}}{r_{i j}^{3}}\left(r_{i}-r_{j}\right),
$$

for $i=1, \ldots, N$. The equations in (2) are called equations of central configurations and are equivalent to the following set of equations (see Hampton and Santoprete 2007)

$$
f_{i j l}=\sum_{k=1, k \neq i, j, l}^{N} m_{k}\left(R_{i k}-R_{j k}\right) \Delta_{i j l k}=0,
$$

for $i<j, l \neq i, l \neq j, i, j, l=1, \ldots, N$, where $R_{i j}=r_{i j}^{-3}$ and $\Delta_{i j l k}=\left(r_{i}-r_{j}\right) \wedge\left(r_{i}-r_{l}\right) \cdot\left(r_{i}-r_{k}\right)$ is six times the oriented volume defined by the tetrahedron with vertices at $r_{i}, r_{j}, r_{l}$ and $r_{k}$.

For the 7-body problem there are 105 equations in (3) which are called Andoyer equations. They are a convenient set of equations to study some classes of central configurations, mainly when there exist symmetries in the configurations.

There exist several symmetries in our configurations (see Figure 1). From the hypotheses of Theorem 1 we have

$$
\begin{gathered}
R_{12}=R_{13}=R_{23}=R_{45}=R_{46}=R_{56}, \\
R_{14}=R_{25}=R_{36}, \\
R_{i 7}=d^{-3}>0, \forall i \in\{1,2, \ldots, 6\},
\end{gathered}
$$

$2 \Delta_{1237}=\Delta_{1234}=\Delta_{1235}=\Delta_{1236}=-\Delta_{4561}=-\Delta_{4562}=-\Delta_{4563}=-2 \Delta_{4567}$, and many others.

Using these symmetries in equations (3), it follows that the equations

$$
\begin{aligned}
& f_{124}=0, \quad f_{125}=0, \quad f_{134}=0, f_{136}=0, f_{235}=0, f_{236}=0, \\
& f_{451}=0, \quad f_{452}=0, \quad f_{461}=0, \quad f_{463}=0, \quad f_{562}=0, \quad f_{563}=0
\end{aligned}
$$

are already verified. Thus, we still have to study the remaining 93 equations.

Consider the equations $f_{123}=0$ and $f_{135}=0$. Using the above symmetries we have

$$
f_{123}=\left(m_{4}-m_{5}\right)\left(R_{14}-R_{24}\right) \Delta_{1234}=0
$$

and

$$
f_{135}=\left(m_{4}-m_{6}\right)\left(R_{14}-R_{34}\right) \Delta_{1354}=0 .
$$


By the hypotheses of Theorem $1, R_{24} \neq R_{14} \neq R_{34}, \Delta_{1234} \neq 0$ and $\Delta_{1354} \neq 0$. So, such equations are satisfied if and only if

$$
m_{4}=m_{5}=m_{6}
$$

Consider also the equations $f_{456}=0$ and $f_{462}=0$. Using the above symmetries we have

$$
f_{456}=\left(m_{1}-m_{2}\right)\left(R_{41}-R_{51}\right) \Delta_{4561}=0
$$

and

$$
f_{462}=\left(m_{1}-m_{3}\right)\left(R_{41}-R_{61}\right) \Delta_{4621}=0 .
$$

By the hypotheses of Theorem $1, R_{51} \neq R_{41} \neq R_{61}, \Delta_{4561} \neq 0$ and $\Delta_{4621} \neq 0$. So, such equations are satisfied if and only if

$$
m_{1}=m_{2}=m_{3} .
$$

Consider now the equation $f_{142}=0$. Using the above symmetries we have

$$
f_{142}=\left(m_{3}-m_{6}\right)\left(R_{13}-R_{43}\right) \Delta_{1423}=0 .
$$

By the hypotheses of Theorem $1, R_{13} \neq R_{43}, \Delta_{1423} \neq 0$. So, such equation is satisfied if and only if

$$
m_{3}=m_{6}
$$

Thus, in order to have a central configuration with the symmetries imposed in Theorem 1, the masses $m_{1}, m_{2}, m_{3}, m_{4}, m_{5}$ and $m_{6}$ must be equal. Item a) of Theorem 1 is proved.

Taking into account $m=m_{1}=m_{2}=m_{3}=m_{4}=m_{5}=m_{6}$ and the symmetries in the hypotheses of Theorem 1, the following equations are already satisfied:

$$
\begin{array}{lllll}
f_{123}=0, & f_{126}=0, & f_{127}=0, & f_{132}=0, & f_{135}=0, \\
f_{137}=0, & f_{142}=0, & f_{143}=0, & f_{145}=0, & f_{146}=0, \\
f_{147}=0, & f_{152}=0, & f_{154}=0, & f_{163}=0, & f_{164}=0, \\
f_{174}=0, & f_{231}=0, & f_{234}=0, & f_{237}=0, & f_{241}=0, \\
f_{245}=0, & f_{251}=0, & f_{253}=0, & f_{254}=0, & f_{256}=0, \\
f_{257}=0, & f_{263}=0, & f_{265}=0, & f_{275}=0, & f_{341}=0, \\
f_{346}=0, & f_{352}=0, & f_{356}=0, & f_{361}=0, & f_{362}=0, \\
f_{364}=0, & f_{365}=0, & f_{367}=0, & f_{376}=0, & f_{453}=0, \\
f_{456}=0, & f_{457}=0, & f_{462}=0, & f_{465}=0, & f_{467}=0, \\
f_{471}=0, & f_{561}=0, & f_{564}=0, & f_{567}=0, & f_{572}=0,
\end{array}
$$

and $f_{673}=0$. Thus, we have 42 equations remaining to analyze.

The 42 remaining equations can be divided into three sets of equivalent equations.

Case 1. The following 12 equations are equivalent:

$$
\begin{aligned}
& f_{153}=0, f_{156}=0, \quad f_{162}=0, f_{165}=0, f_{243}=0, f_{246}=0, \\
& f_{261}=0, \quad f_{264}=0, \quad f_{342}=0, f_{345}=0, \quad f_{351}=0, f_{354}=0 \text {. }
\end{aligned}
$$


Thus it is sufficient to study only one of these equations, for instance, the equation $f_{153}=0$ which can be written as

$$
\left(3 R_{12}-2 R_{52}-R_{53}\right) \Delta_{1532}=0 .
$$

Case 2. The following 6 equations are equivalent:

$$
f_{157}=0, \quad f_{167}=0, \quad f_{247}=0, \quad f_{267}=0, \quad f_{347}=0, \quad f_{357}=0 .
$$

Thus it is sufficient to study only one of these equations, for instance, the equation $f_{157}=0$ which can be written as

$$
\left(3 R_{12}-2 R_{52}-R_{53}\right) \Delta_{1572}=0 .
$$

Case 3. The following 24 equations are equivalent

$$
\begin{aligned}
& f_{172}=0, \quad f_{173}=0, \quad f_{175}=0, \quad f_{176}=0, \quad f_{271}=0, \quad f_{273}=0, \\
& f_{274}=0, \quad f_{276}=0, \quad f_{371}=0, \quad f_{372}=0, \quad f_{374}=0, \quad f_{375}=0 \text {, } \\
& f_{472}=0, \quad f_{473}=0, \quad f_{475}=0, f_{476}=0, f_{571}=0, \quad f_{573}=0 \text {, } \\
& f_{574}=0, \quad f_{576}=0, \quad f_{671}=0, \quad f_{672}=0, \quad f_{674}=0, \quad f_{675}=0 .
\end{aligned}
$$

Thus it is sufficient to study only one of these equations, for instance, the equation $f_{172}=0$ which can be written as

$$
\left(3 R_{12}-2 R_{52}-R_{53}\right) \Delta_{1723}=0 .
$$

Under our hypotheses the terms $\Delta_{1532}, \Delta_{1572}$ and $\Delta_{1723}$ do not vanish, so equations (4), (5) and (6) are satisfied if and only if

$$
3 R_{12}-2 R_{52}-R_{53}=0 .
$$

Equation (7) implies that the central configurations studied here do not depend on the value of the mass $m_{7}$.

In order to simplify our analysis and without loss of generality, take a system of coordinates in which $r_{i}=\left(x_{i}, y_{i}, z_{i}\right) \in \mathbb{R}^{3}$, where

$$
\begin{gathered}
r_{1}=(x, 0, y), \quad r_{2}=\left(\frac{-x}{2}, \frac{\sqrt{3} x}{2}, y\right), \quad r_{3}=\left(\frac{-x}{2},-\frac{\sqrt{3} x}{2}, y\right), \\
r_{4}=(x, 0,-y), \quad r_{5}=\left(\frac{-x}{2}, \frac{\sqrt{3} x}{2},-y\right), \quad r_{6}=\left(\frac{-x}{2},-\frac{\sqrt{3} x}{2},-y\right), \\
r_{7}=(0,0,0), \quad x>0, \quad y>0 .
\end{gathered}
$$

With these coordinates $x=a>0$ and $2 y>0$ is the distance between the planes $\Pi_{1}$ and $\Pi_{2}$. It follows that equation (7) is written as

$$
F(x, y)=\frac{3}{(\sqrt{3} x)^{3}}-\frac{2}{(2 y)^{3}}-\frac{1}{\left(3 x^{2}+4 y^{2}\right)^{3 / 2}}=0
$$

with $x>0$ and $y>0$.

Therefore, to complete the proof of Theorem 1 we need to study the zero level of the function $F$ in (8). We claim that the zero level of $F$ is contained in a straight line passing through the origin. In fact, consider the change of variables defined by

$$
u=\sqrt{3} x, \quad v=2 y \text {. }
$$


With these new variables the function $F$ in (8) is written as

$$
F(u, v)=\frac{3}{u^{3}}-\frac{2}{v^{3}}-\frac{1}{\left(u^{2}+v^{2}\right)^{3 / 2}}
$$

Now, taking polar coordinates

$$
u=r \cos \theta, v=r \sin \theta
$$

the function $F$ is given by

$$
F(r, \theta)=\frac{1}{r^{3}}\left[\frac{3}{\cos ^{3} \theta}-\frac{2}{\sin ^{3} \theta}-1\right]=\frac{1}{r^{3}}\left[\frac{3 \sin ^{3} \theta-2 \cos ^{3} \theta-\sin ^{3} \theta \cos ^{3} \theta}{\sin ^{3} \theta \cos ^{3} \theta}\right] .
$$

From (10) the zero level of $F$ is obtained from the zeros of the function

$$
3 \sin ^{3} \theta-2 \cos ^{3} \theta-\sin ^{3} \theta \cos ^{3} \theta=\cos ^{3} \theta\left(3 \tan ^{3} \theta-\sin ^{3} \theta-2\right),
$$

that is, from the function

$$
f(\theta)=3 \tan ^{3} \theta-\sin ^{3} \theta-2, \quad \theta \in\left(0, \frac{\pi}{2}\right) .
$$

From elementary calculations we have

$$
\lim _{\theta \rightarrow 0+} f(\theta)=-2<0, \quad \lim _{\theta \rightarrow \frac{\pi}{2}^{-}} f(\theta)=+\infty, \quad f^{\prime}(\theta)=\frac{3 \sin ^{2} \theta}{\cos ^{4} \theta}\left(3-\cos ^{5} \theta\right)>0,
$$

for all $\theta \in(0, \pi / 2)$. Thus, $f$ is an increasing function that changes sign only once. Therefore, there is only one $\theta_{0} \in(0, \pi / 2)$ such that $f\left(\theta_{0}\right)=0$. This implies that the zero level of $F$ is contained in the set $\left\{\theta_{0}, r>0\right\}$, that is the zero level of $F$ is given by the following set

$$
\mathcal{Z}=\left\{(x, y) \in \mathbb{R}^{2}: x>0, y=\alpha x\right\},
$$

for some $\alpha>0$ in the original coordinates. Simple numerical computations give the approximated value $\alpha \simeq 0.7935817272$.

The uniqueness of the class of central configuration studied here follows from the set $\mathcal{Z}$ in (12). This end the proof of Theorem 1 .

\section{CONCLUSIONS}

An interesting fact about the configuration studied here is that it does not depend on the values of the masses $m$ and $m_{7}$. So we have a unique two parameter class of central configurations. Also, if we remove the body of mass $m_{7}$ the remaining six bodies are already in a central configuration (see Cedó and Llibre 1989). Thus the central configuration studied here is an example of spatial stacked central configuration with seven bodies (see Hampton and Santoprete 2007, Mello et al. 2009).

The results obtained in this paper also work for other regular $n$-gons instead the equilateral triangle, but this is a subject of a future work. At the moment we have just numerical results.

We believe that the results obtained are true for the case where one triangle is rotated by an angle of $\pi / 3$ with respect to the other one. Rotations by other angles require an approach different of the presented here and new techniques must be found. 
We also believe that similar results can be obtained taking the same structure with two equal co-circular central configurations (see Cors and Roberts 2012), instead of two equilateral triangles.

\section{ACKNOWLEDGMENTS}

The authors were partially supported by Fundação de Amparo à Pesquisa do Estado de Minas Gerais (FAPEMIG) grant APQ-00015/12. The second author was partially supported by Conselho Nacional de Desenvolvimento Científico e Tecnológico (CNPq) grant 301758/2012-3 and by FAPEMIG grant PPM00092-13.

\section{RESUMO}

Neste artigo estudamos a existência de novas famílias de configurações centrais espaciais para o problema de 7 corpos. Nas configurações estudadas aqui seis corpos estão nos vértices de dois triângulos equiláteros $\mathcal{T}_{1}, \mathcal{T}_{2}$ e um corpo está localizado fora dos planos paralelos distintos contendo $\mathcal{T}_{1}$ e $\mathcal{T}_{2}$. Os resultados apresentados aqui tem provas simples e analíticas.

Palavras-chave: configuração espacial, problema de 7 corpos, configuração central, configuração central empilhada.

\section{REFERENCES}

Cedó F AND LliBRE J. 1989. Symmetric central configurations of the spatial n-body problem. J Geom Phys 6: 367-394.

Corbera M AND Llibre J. 2008. Central configurations of nested regular polyhedra for the spatial $2 n$-body problem. J Geom Phys 58: 1241-1252.

CORBERA M AND Llibre J. 2009. Central configurations of three regular polyhedra for the spatial $3 n$-body problem. J Geom Phys 59: 321-339.

CORS JM AND RoBERTS G. 2012. Four-body co-circular central configurations. Nonlinearity 25: 343-370.

Hagihara Y. 1970. Celestial Mechanics, vol. 1, Massachusetts: MIT Press, 720 p.

HAMPTON M AND SANTOPRETE M. 2007. Seven-body central configurations: a family of central configurations in the spatial sevenbody problem. Celestial Mech Dyn Astr 99: 293-305.

LEHMANN-FiLHÉS R. 1891. Ueber zwei Fälle des Vielkörperproblems. Astr Naschr 127: 137-144.

Mello LF, Chaves FE, Fernandes AC And Garcia BA. 2009. Stacked central configurations for the spatial 6-body problem. J Geom Phys 59: 1216-1226.

MELlo LF AND FERNANDES AC. 2011a. New classes of spatial central configurations for the $n+3$-body problem. Nonlinear Anal Real World Appl 12: 723-730.

MELlo LF AND FERNANDES AC. 2011b. New spatial central configurations in the 5-body problem. An Acad Bras Cienc 83: $763-774$. MoECKEL R. 1990. On central configurations. Math Z 205: 499-517.

NeWton I. 1687. Philosophi Naturalis Principia Mathematica. London: Royal Society, 512 p.

SAARI D. 1980. On the role and properties of central configurations. Celestial Mech 21: 9-20.

SMALE S. 1970. Topology and mechanics II: The planar n-body problem. Invent Math 11: 45-64.

Wintner A. 1941. The Analytical Foundations of Celestial Mechanics. Princeton: Princeton University Press, 448 p.

ZHANG S AND ZHOU Q. 2001. Double pyramidal central configurations. Phys Lett A 281: 240-248.

ZHU C. 2005. Central configurations of nested regular tetrahedrons. J Math Anal Appl 312: 83-92. 